\title{
Mora: ¿ Fuente De Ingresos Para Productores $Y$ Comercializadores?. Un Enfoque Desde El Análisis De La Dinámica Del Mercado De Las Familias De La Ciudad De Riobamba. Estudio De Caso: Parroquia El Altar, Ecuador.
}

\author{
Gabriela Natalí Fonseca Romero \\ Danny Daniel Castillo Vizuete \\ Luis Alberto Quevedo Báez
}

Investigadores, Escuela Superior Politécnica de Chimborazo.

\section{doi: 10.19044/esj.2017.v13n7p399 URL:http://dx.doi.org/10.19044/esj.2017.v13n7p399}

\begin{abstract}
Agriculture is one of the main economic activities in Ecuador, and it is fundamental to an economy in terms of food security. Currently, the country is involved in a process of changing its production system. Its main objective is to carry out the substitution of exports for imports and the generation of added value. In order to achieve these goals, agriculture is considered as a dynamic element of the rural Andean regions. El Altar is a rural parish located in the Penipe area of Chimborazo Province. Due to its particular geographical location, El Altar has several climates ranging from the subtropical to the high altitude plane. This climate facilitates the development of a heterogeneous agriculture. In this mix, the cultivation of blackberries has developed into an important economy activity for the parish inhabitants. Consequently, based on an analysis of the demand of the competition and a calculation of the objective demand, we determined the likely acceptance of families in Riobamba to consume the blackberries cultivated in the El Altar parish. Also, we ascertain the possibility of the producers to expand their market and the profit the seller could receive. In conclusion, it was found that families in Riobamba would like to consume the blackberry cultivated from El Altar. However, this activity could generate significant economic benefits for the producers and commercial sellers of this fruit.
\end{abstract}

Keywords: Blackberry farmers, blackberry sellers, rural development, market study, farming, Chimborazo, Ecuador 


\section{Resumen}

El Ecuador es un país considerado eminentemente agrícola, dicha actividad es fundamental en el ámbito económico, como en la seguridad alimentaria. Actualmente el Ecuador atraviesa un cambio de la "matriz productiva", donde su objetivo primordial es la sustitución de importaciones por exportaciones y la generación de valor agregado, dentro de la cual está inmersa la agricultura, como elemento dinamizador de territorios rurales andinos. El Altar es una parroquia rural del cantón Penipe, provincia de Chimborazo, la misma que debida a su ubicación geográfica, posee varios climas desde el subtropical hasta el frío del páramo, lo cual ha permitido el desarrollo de la agricultura en forma amplia, siendo el cultivo de "mora" una actividad importante a la cual se dedican los habitantes de la parroquia. Es así que, a partir del análisis de la demanda, análisis de la competencia y cálculo de la demanda objetiva; se determina la aceptación que tienen las familias de Riobamba para consumir la mora de la parroquia El Altar, la posibilidad que tienen los productores de la parroquia para ampliar el mercado y la rentabilidad que obtiene el vendedor al comercializar este producto. En conclusión, la mora de la parroquia El Altar es apetecible para las familias de Riobamba. Se constituye en una fuente de ingresos para los productores y comercializadores de este fruto.

Palabras claves: Productores de mora, comercializadores de mora, desarrollo rural, estudio de mercado, agricultura, Chimborazo, Ecuador

\section{Introducción}

La pobreza de acuerdo con Spicker (2009), se toma desde 4 puntos de vista: desde lo material, se asocia directamente con falta de recursos para alcanzar un nivel de vida digno, en ella incluye conceptos como necesidad, patrón de privaciones y limitación de recursos; desde lo económico, la pobreza se mide con el ingreso asociando términos como nivel de vida, desigualdad y posición económica; desde las condiciones sociales, lo cual se centra en la forma de vida de la población de acuerdo a la clase social, el grado de dependencia, la carencia de seguridad básica, la ausencia de titularidades y la exclusión y finalmente desde el juicio moral, en el cual las personas son pobres cuando se juzga que sus condiciones materiales son moralmente inaceptables. Echeverría (2011), manifiesta que la pobreza rural es más aguda que la urbana y parece más difícil de superarla. En este sentido, Zegarra y Tuesta (2009), afirma que el crecimiento agrícola y su relación con este apartado es que la desigualdad del ingreso de la población rural no ha mejorado sino lo contrario. Por su parte Klein (2012), sostiene que la pobreza rural se genera por la informalidad y la precariedad en el empleo. 
El sector pobre de la economía del mundo se encuentra en las zonas rurales quienes conforman las tres cuartas partes de los mil doscientos millones de pobres existentes en el mundo (IFAD, 2001). Actualmente en América Latina, el Ecuador no es la excepción y se han venido desarrollando varios modelos de desarrollo rural Castillo y Quevedo (2016). En este sentido, el Ecuador se enfoca en un modelo de desarrollo incluyente y sostenible llamado "El Buen Vivir", que se centra en el bienestar y los derechos humanos de los ciudadanos, fundamentado en 4 variables: económico, ambiental, social y cultural Castillo y Quevedo (2016). Por otro lado Formichella y Massigoge (2004), manifiestan que el desarrollo local de un territorio se propicia al aumentar las cualidades emprendedoras de la comunidad, aumentando así la aparición de nuevas e innovadoras iniciativas en la misma, facilitando la tarea de los agentes de desarrollo y favoreciendo así la generación de proyectos y la formación de redes sociales. Es así que, el desarrollo debe ser concebido desde una visión holística que integre múltiples aristas, generando capacidades en los pobladores locales que les permita realmente tener alternativas de mejora ancladas al Buen Vivir.

La economía de países latinoamericanos depende en gran medida de la producción y comercialización de productos agrícolas entre los cuales se destacan las frutas y hortalizas (Ayala et al., 2013). En el Ecuador el cultivo de mora tiene un gran potencial, dadas las características agro ecológicas y climáticas, aptas para la explotación de esta especie y con una producción en todos los meses del año, cuya particularidad otorga ventaja sobre los mayores productores a nivel mundial (Morillo, 2011). Por ser una fruta de consumo diario de las familias ecuatorianas, su demanda es alta especialmente en la costa, para la exportación de la mora de castilla se debe tomar en cuenta que en varios países se exige requisitos que debe reunir el fruto, como ausencia de residuos de pesticidas, empaque adecuado y una excelente presentación (MAGAP, 2013).

Bajo este contexto el presente artículo tiene como objetivo analizar la demanda, analizar la competencia y calcular la demanda objetiva, para determinar la aceptación que tienen las familias de Riobamba para consumir la mora de la parroquia El Altar, la posibilidad que tienen los productores de la parroquia para ampliar el mercado y la rentabilidad que obtiene el vendedor al comercializar este producto.

\section{Metodología}

\subsection{Análisis de la demanda}

La población de estudio considerada para la presente investigación está contemplada por las familias de estrato medio y bajo de la ciudad de Riobamba. Esta población se consideró debido a que Instituto Nacional de 
Estadísticas y Censos (INEC, 2011), manifiesta que el nivel socioeconómico alto (nivel A) realiza sus compras en centros comerciales.

De acuerdo a los datos obtenidos a través del INEC (2010), en la ciudad de Riobamba existen 162.575 habitantes, tomando en cuenta el promedio de personas por hogar publicado por el INEC (2010) que es de 3,78 tenemos un total de 43.000 familias, de las cuales según la encuesta de estratificación el 83,3\% corresponde a un nivel socioeconómico medio (nivel B, C+, C-), y el 14,9 \% corresponden a un estrato bajo (nivel D) existiendo por lo tanto 42.226 familias. Es así que se estableció la muestra, misma que fue calculada con un error del $8 \%$ y con un valor de confianza igual a 2 , con una probabilidad de ocurrencia y no ocurrencia de $50 \%$, obteniendo como resultado 156 personas a ser encuestadas.

La técnica empleada fue la encuesta que permitió la recolección de la información y el instrumento utilizado para recabar información fue el cuestionario.

\subsection{Análisis de la competencia}

Para definir la competencia se consideró los productores de mora que comercializan su producto en el mercado mayorista. La técnica empleada fue la entrevista que permitió la recolección de la información y el instrumento utilizado para recabar información fue la guía de entrevista.

\subsection{Determinación de la demanda total, potencial, insatisfecha y objetiva}

La demanda total es el universo de estudio (DT=U), la demanda potencial fue obtenida mediante la ecuación: (DP=DT*AM), es decir la relación entre la demanda total y el porcentaje de aceptación del mercado. Así también la demanda insatisfecha resulta del cálculo entre la demanda potencial y la diferencia de la competencia (DI=DP-C). Mientras que la demanda objetiva se calculó entre al demanda insatisfecha y el porcentaje de captación del mercado $\left(\mathrm{DP}=\mathrm{DI}^{*} \mathrm{AC}\right)$. Las proyecciones se realizaron utilizando el método de incremento compuesto cuyo modelo matemático es: $C_{n}=\mathrm{C}_{\mathrm{o}}(1+i)^{\mathrm{n}}$. El consumo aparente por familias se determinó de la división de la demanda objetiva en períodos de tiempo.

\section{Resultados y discusión}

\subsection{Análisis de la demanda}

\section{Preferencia de consumo de mora}

Cuadro 1. Preferencia de consumo de mora

\begin{tabular}{|c|c|c|}
\hline Opciones & Frecuencia Absoluta & Frecuencia Relativa \\
\hline $\mathrm{Si}$ & 144 & 92 \\
\hline No & 12 & 8 \\
\hline TOTAL & $\mathbf{1 5 6}$ & $\mathbf{1 0 0}$ \\
\hline
\end{tabular}


Conocimiento de consumo de mora producida en la parroquia El Altar

Cuadro 2. Conocimiento de consumo de mora producida en la parroquia El Altar

\begin{tabular}{|c|c|c|}
\hline Opciones & Frecuencia Absoluta & Frecuencia Relativa \\
\hline $\mathrm{Si}$ & 13 & 8 \\
\hline No & 31 & 20 \\
\hline Desconoce & 112 & 72 \\
\hline TOTAL & $\mathbf{1 5 6}$ & $\mathbf{1 0 0}$ \\
\hline
\end{tabular}

Aceptación de compra de la mora producida en la parroquia El Altar

Cuadro 3. Aceptación de compra de la mora producida en la parroquia El Altar

\begin{tabular}{|c|c|c|}
\hline Opciones & Frecuencia Absoluta & Frecuencia Relativa \\
\hline $\mathrm{Si}$ & 137 & 88 \\
\hline $\mathrm{No}$ & 19 & 12 \\
\hline TOTAL & $\mathbf{1 5 6}$ & $\mathbf{1 0 0}$ \\
\hline
\end{tabular}

DT= Demanda Total; U= Universo de estudio; $\mathrm{DP}=$ Demanda Potencial; $\mathrm{AM}=\%$ de aceptación del mercado; DI= Demanda Insatisfecha; C= Competencia; $\mathrm{DO}=$ Demanda Objetiva; $\mathrm{CM}=\%$ Captación del mercado.

$\mathrm{C}_{\mathrm{n}}=$ clientes proyectados; $\mathrm{C}_{\mathrm{o}}=$ clientes iniciales (demanda objetiva inicial); $\mathrm{i}=$ incremento de la población; $\mathrm{n}=$ año proyectado.

Frecuencia de consumo de mora

Cuadro 4. Frecuencia de consumo de mora

\begin{tabular}{|l|l|l|}
\hline Opciones & Frecuencia Absoluta & Frecuencia Relativa \\
\hline Diario & 8 & 5 \\
\hline Semanal & 47 & 30 \\
\hline Quincenal & 78 & 50 \\
\hline Mensual & 23 & 15 \\
\hline TOTAL & $\mathbf{1 5 6}$ & $\mathbf{1 0 0}$ \\
\hline
\end{tabular}

Cantidad de consumo semanal de mora

Cuadro 5. Cantidad de consumo semanal de mora

\begin{tabular}{|c|c|c|}
\hline Opciones & Frecuencia Absoluta & Frecuencia Relativa \\
\hline Menos de $1 \mathrm{Kg}$. & 98 & 63 \\
\hline $1-2 \mathrm{Kg}$. & 42 & 27 \\
\hline 3-4 Kg. & 14 & 9 \\
\hline más de 4 Kg. & 2 & 1 \\
\hline TOTAL & $\mathbf{1 5 6}$ & $\mathbf{1 0 0}$ \\
\hline
\end{tabular}

\section{Precio de libra de mora}

Cuadro 6. Precio de libra de mora

\begin{tabular}{|c|c|c|}
\hline Opciones & Frecuencia Absoluta & Frecuencia Relativa \\
\hline$\$ 1$ & 80 & 51 \\
\hline$\$ 1,25$ & 68 & 44 \\
\hline$\$ 1,50$ & 8 & 5 \\
\hline TOTAL & $\mathbf{1 5 6}$ & $\mathbf{1 0 0}$ \\
\hline
\end{tabular}




\subsubsection{Discusión}

La mora al tratarse de un fruto que se caracteriza principalmente por su sabor ácido tiene una gran aceptación en el mercado, un 92\% de los compradores gustan del consumo de esta fruta (ver cuadro 1). Con relación a la comercialización de mora de la parroquia El Altar, los consumidores en un $72 \%$ desconocen que la mora que compran proceda de dicho lugar (ver cuadro 2), pero el $88 \%$ de los mismos les gustaría consumir la mora que se produce en la parroquia (ver cuadro 3). La mayoría de hogares riobambeños (ver cuadro 4) consumen este fruto con una frecuencia quincenal y lo hacen con una cantidad de consumo menos a $1 \mathrm{Kg}$. por semana (ver cuadro 5). En lo referente al precio de la libra de mora un $51 \%$ paga un valor de $\$ 1$, mientras un $44 \%$ pagaría hasta $\$ 1,25$. 


\subsection{Análisis de la competencia}

Cuadro 7. Análisis de la competencia

\begin{tabular}{|c|c|c|c|c|c|c|c|c|c|c|c|}
\hline NOMBRE & $\begin{array}{c}\text { PRECIO } \\
\text { AL QUE } \\
\text { VENDEN } \\
\text { LA CAJA } \\
\text { DE } \\
\text { MORA }\end{array}$ & $\begin{array}{c}\text { VARIEDAD } \\
\text { DE MORA } \\
\text { QUE } \\
\text { CULTIVAN }\end{array}$ & $\begin{array}{c}\text { HECTÁR } \\
\text { EAS DE } \\
\text { CULTIVO } \\
\text { DE } \\
\text { MORA }\end{array}$ & $\begin{array}{c}\text { JORNALES } \\
\text { POR } \\
\text { HECTÁRE } \\
\text { A QUE } \\
\text { CONTRAT } \\
\text { AN PARA } \\
\text { LAS } \\
\text { ACTIVIDA } \\
\text { DES DE } \\
\text { CULTIVO }\end{array}$ & $\begin{array}{c}\text { RECIPIENTE } \\
\text { S QUE } \\
\text { UTILIZAN } \\
\text { PARA } \\
\text { COSECHAR } \\
\text { LA MORA }\end{array}$ & $\begin{array}{c}\text { ¿EFECTÚA } \\
\text { ALGUN } \\
\text { TIPO DE } \\
\text { ALMACEN } \\
\text { AMIENTO } \\
\text { DESPUES } \\
\text { DE LA } \\
\text { COSECHA? }\end{array}$ & $\begin{array}{c}\dot{¿}^{A} \text { QUUIEN } \\
\text { VENDE SU } \\
\text { PRODUCCIÓ } \\
\text { N? }\end{array}$ & $\begin{array}{c}\text { ÉPOCA DE } \\
\text { MAYOR } \\
\text { PRODUCCI } \\
\text { ON }\end{array}$ & $\begin{array}{c}\text { FERTILIZAC } \\
\text { IÓN } \\
\text { UTILIZADA } \\
\text { PARA EL } \\
\text { CULTIVO }\end{array}$ & $\begin{array}{c}\text { NÚMERO } \\
\text { DE CAJAS } \\
\text { ENTREGA } \\
\text { DAS A LOS } \\
\text { VENDEDO } \\
\text { RES } \\
\text { SEMANAL } \\
\text { MENTE }\end{array}$ & $\begin{array}{c}\text { NÚMERO DE } \\
\text { FAMILIAS A } \\
\text { LAS QUE } \\
\text { VENDEN LA } \\
\text { MORA } \\
\text { ANUALMEN } \\
\text { TE }\end{array}$ \\
\hline SAN LUIS & $\$ 6,00$ & $\begin{array}{c}\text { Castilla con } \\
\text { espino/Castill } \\
\text { a sin espino }\end{array}$ & 80 & 3 & $\begin{array}{c}\text { Caja de } \\
\text { madera/Tarrina }\end{array}$ & $\mathrm{Si}$ & Intermediarios & $\begin{array}{l}\text { Octubre- } \\
\text { Diciembre }\end{array}$ & $\begin{array}{l}\text { Mixta(químico } \\
\text {-orgánico) }\end{array}$ & 50 & 7200 \\
\hline CHILLANES & $\$ 5,50$ & $\begin{array}{c}\text { Castilla con } \\
\text { espino/Castill } \\
\text { a sin espino }\end{array}$ & 50 & 3 & Balde & No & Intermediarios & Enero-Marzo & $\begin{array}{l}\text { Mixta(químico } \\
\text {-orgánico) }\end{array}$ & 35 & 5040 \\
\hline BAYUSHIG & $\$ 6,00$ & $\begin{array}{c}\text { Castilla sin } \\
\text { espino }\end{array}$ & 40 & 3 & Caja de madera & No & Intermediarios & Enero-Marzo & $\begin{array}{l}\text { Mixta(químico } \\
\text {-orgánico) }\end{array}$ & 30 & 4320 \\
\hline MATUS & $\$ 6,00$ & $\begin{array}{c}\text { Castilla sin } \\
\text { espino }\end{array}$ & 30 & 3 & Caja de madera & No & Intermediarios & Enero-Marzo & $\begin{array}{c}\text { Mixta(químico } \\
\text {-orgánico) }\end{array}$ & 25 & 3600 \\
\hline BILBAO & $\$ 6,00$ & $\begin{array}{c}\text { Castilla sin } \\
\text { espino }\end{array}$ & 15 & 2 & Caja de madera & No & $\begin{array}{l}\text { Consumidor } \\
\text { final }\end{array}$ & Enero-Marzo & $\begin{array}{l}\text { Mixta(químico } \\
\text {-orgánico) }\end{array}$ & 20 & 2880 \\
\hline QUIMIAG & $\$ 5,80$ & $\begin{array}{c}\text { Castilla con } \\
\text { espino }\end{array}$ & 20 & 2 & $\begin{array}{c}\text { Caja de } \\
\text { madera/Tarrina }\end{array}$ & Si & $\begin{array}{c}\text { Consumidor } \\
\text { final }\end{array}$ & $\begin{array}{c}\text { Octubre- } \\
\text { Diciembre }\end{array}$ & $\begin{array}{l}\text { Mixta(químico } \\
\text {-orgánico) }\end{array}$ & 20 & 2880 \\
\hline LICTO & $\$ 6,00$ & \begin{tabular}{|c|} 
Castilla con \\
espino/Castill \\
a sin \\
espino/Gato \\
\end{tabular} & 20 & 3 & Caja de madera & No & Intermediarios & $\begin{array}{l}\text { Octubre- } \\
\text { Diciembre }\end{array}$ & $\begin{array}{l}\text { Mixta(químico } \\
\text {-orgánico) }\end{array}$ & 20 & 2880 \\
\hline \multicolumn{12}{|c|}{ TOTAL } \\
\hline
\end{tabular}




\subsubsection{Discusión}

$\mathrm{Al}$ analizar la competencia y las variables expuestas (ver cuadro 7), referente al precio se puede argumentar que no todos los competidores entregan al mismo valor la caja de mora siendo una de las razones la calidad de mora que entregan. Por ejemplo, Chillanes entrega a $\$ 11$ el balde, el mismo que contiene 2 cajas de madera es decir $6 \mathrm{~kg}$, equivalente a $\$ 5,50$ cada caja, el valor más bajo en relación al resto de competidores, esto se debe a que los productores de Chillanes utilizan como recipientes para el cultivo de mora baldes y no cajas, esto hace que la mora sufra un maltrato por la manipulación y además, otro factor que influye en la calidad de la mora es la distancia existente Riobamba - Chillanes, ya que la mora permanece en baldes aproximadamente 3 horas de viaje expuesta a condiciones que afectan la calidad del fruto, todo esto hace que la mora tienda a madurarse con mayor rapidez. Las variedades de mora cumplen un papel muy importante que tiene estrecha relación con el precio y la calidad del fruto, debido a que los productores que cultivan mora de castilla sin espino, de acuerdo al Instituto Nacional de Investigaciones Agropecuarias (INIAP, 2013), esta variedad es de fácil manejo, pues se puede manipular con las manos sin protección y sin dañar el fruto al momento de la cosecha. Esto permite que los productores entreguen al intermediario y por ende al consumidor final un producto en condiciones favorables para el consumo.

En este contexto, es importante realizar un análisis particular del precio de venta del productor y el vendedor, mismo que se lo presenta a continuación:

Cuadro 8. Análisis de precios productor

\begin{tabular}{|c|c|c|c|}
\hline \multicolumn{4}{|c|}{ PRODUCTOR } \\
\hline $\begin{array}{c}\text { Fluctuación Precios/ } \\
\text { caja }\end{array}$ & Kg/caja & Lbs/caja & $\begin{array}{c}\text { Precio libra que } \\
\text { entrega el productor }\end{array}$ \\
\hline$\$ 6,00$ & 3 & 6,6 & $\$ 0,91$ \\
\hline$\$ 5,80$ & 3 & 6,6 & $\$ 0,88$ \\
\hline$\$ 5,50$ & 3 & 6,6 & $\$ 0,83$ \\
\hline
\end{tabular}

Cuadro 9. Análisis de precios vendedor

\begin{tabular}{|c|c|c|}
\hline \multicolumn{3}{|c|}{ VENDEDOR } \\
\hline Precio de Venta & Precio libra productor & Utilidad por libra \\
\hline$\$ 1,00$ & $\$ 0,91$ & $\$ 0,09$ \\
\hline$\$ 1,00$ & $\$ 0,88$ & $\$ 0,12$ \\
\hline$\$ 1,00$ & $\$ 0,83$ & $\$ 0,17$ \\
\hline$\$ 1,25$ & $\$ 0,91$ & $\$ 0,34$ \\
\hline$\$ 1,25$ & $\$ 0,88$ & $\$ 0,37$ \\
\hline$\$ 1,25$ & $\$ 0,83$ & $\$ 0,42$ \\
\hline$\$ 1,50$ & $\$ 0,91$ & $\$ 0,59$ \\
\hline$\$ 1,50$ & $\$ 0,88$ & $\$ 0,62$ \\
\hline$\$ 1,50$ & $\$ 0,83$ & $\$ 0,67$ \\
\hline
\end{tabular}


Cuadro 10. Análisis promedio entre productores y vendedores

\begin{tabular}{|c|c|c|c|c|c|c|c|c|c|c|}
\hline $\begin{array}{l}\text { Preci } \\
\text { o } \\
\text { Prom } \\
\text { edio } \\
\text { por } \\
\text { caja }\end{array}$ & $\begin{array}{c}\mathrm{Kg} / \mathrm{c} \\
\text { aja }\end{array}$ & $\begin{array}{l}\text { Lbs/ } \\
\text { caja }\end{array}$ & $\begin{array}{c}\text { Precio } \\
\text { por } \\
\text { libra } \\
\text { promed } \\
\text { io que } \\
\text { entrega } \\
\text { el } \\
\text { product } \\
\text { or } \\
\end{array}$ & $\begin{array}{c}\text { Pre } \\
\text { cio } \\
\text { de } \\
\text { vent } \\
\text { a }\end{array}$ & $\begin{array}{c}\text { Frec } \\
\text { uenci } \\
\text { a } \\
\text { relati } \\
\text { va } \\
\text { preci } \\
\text { o de } \\
\text { mora }\end{array}$ & $\begin{array}{c}\text { cajas } \\
\text { recibi } \\
\text { das } \\
\text { prome } \\
\text { dio/ } \\
\text { seman } \\
\text { a }\end{array}$ & $\begin{array}{c}\text { libras } \\
\text { recibi } \\
\text { das } \\
\text { prome } \\
\text { dio/ } \\
\text { seman } \\
\text { a }\end{array}$ & $\begin{array}{c}\text { Utilid } \\
\text { ad/lib } \\
\text { ra }\end{array}$ & $\begin{array}{c}\text { utilidad/ } \\
\text { cajas } \\
\text { promedi } \\
\text { o } \\
\text { semanal } \\
\text { de los } \\
\text { vendedor } \\
\text { es }\end{array}$ & $\begin{array}{c}\% \\
\text { utilidad } \\
\text { por cada } \\
\text { caja de } \\
\text { mora }\end{array}$ \\
\hline \multirow{3}{*}{$\$ 5,77$} & \multirow{3}{*}{3} & \multirow{3}{*}{6,6} & \multirow{3}{*}{$\$ 0,87$} & $\begin{array}{c}\$ \\
1,00\end{array}$ & 51 & \multirow{3}{*}{29} & \multirow{3}{*}{189} & $\begin{array}{c}\$ \\
0,13\end{array}$ & $\begin{array}{c}\$ \\
23,81\end{array}$ & 14 \\
\hline & & & & $\begin{array}{c}\$ \\
1,25\end{array}$ & 44 & & & $\begin{array}{c}\$ \\
0,38\end{array}$ & $\begin{array}{c}\$ \\
70,95\end{array}$ & 42 \\
\hline & & & & $\begin{array}{c}\$ \\
1,50\end{array}$ & 5 & & & $\begin{array}{c}\$ \\
0,63\end{array}$ & $\begin{array}{c}\$ \\
118,10\end{array}$ & 69 \\
\hline
\end{tabular}

Se realiza un análisis de precios a los que el productor entrega la caja de mora (ver cuadro 8), teniendo precios fluctuantes desde $\$ 5,50$ a $\$ 6,00$, tomando en cuenta que cada caja contiene $3 \mathrm{Kg}$. que equivale a 6,6 libras, obtenemos el precio de cada libra de mora al cual el productor deja al vendedor. A su vez en el análisis de precios del vendedor (ver cuadro 9) podemos identificar cual es la utilidad por libra de mora que ellos obtienen tomando como referencia los precios que están dispuestos a pagar los consumidores (ver cuadro 6) con los precios que el productor entrega. En el cuadro 10 se realiza un análisis con un precio promedio por caja de mora de los productores y un número promedio de cajas de mora a la semana de los vendedores, obteniendo una utilidad promedio que el comercializador adquiere. Es por esto que se puede concluir que la comercialización de la mora es rentable.

\subsection{Determinación de la demanda total, potencial, insatisfecha y objetiva}

Cuadro 11. Cálculo de la demanda total, potencial, insatisfecha, objetiva y sus proyecciones

\begin{tabular}{|c|c|c|c|c|c|c|}
\hline $\mathbf{N}^{\mathbf{o}}$ & Año & $\begin{array}{c}\text { Demanda } \\
\text { Total }\end{array}$ & $\begin{array}{c}\text { Demanda } \\
\text { Potencial }\end{array}$ & Competencia & $\begin{array}{c}\text { Demanda } \\
\text { Insatisfecha }\end{array}$ & $\begin{array}{c}\text { Demanda } \\
\text { Objetiva }\end{array}$ \\
\hline 0 & 2017 & 42226 & 37159 & 28800 & 8359 & 6687 \\
\hline 1 & 2018 & 42902 & 37753 & 29261 & 8493 & 6794 \\
\hline 2 & 2019 & 43588 & 38357 & 29729 & 8629 & 6903 \\
\hline 3 & 2020 & 44285 & 38971 & 30205 & 8767 & 7013 \\
\hline 4 & 2021 & 44994 & 39595 & 30688 & 8907 & 7125 \\
\hline 5 & 2022 & 45714 & 40228 & 31179 & 9049 & 7239 \\
\hline
\end{tabular}




\section{Consumo aparente por cliente}

Cuadro 12. Consumo aparente por cliente

\begin{tabular}{|c|c|c|c|c|c|c|c|}
\hline $\mathbf{N}^{\mathbf{0}}$ & Año & Semestre & Trimestre & Mes & Quincena & Semana & Día \\
\hline 0 & 2017 & 3344 & 1672 & 557 & 279 & 139 & 18 \\
\hline 1 & 2018 & 3397 & 1699 & 566 & 283 & 142 & 19 \\
\hline 2 & 2019 & 3451 & 1726 & 575 & 288 & 144 & 19 \\
\hline 3 & 2020 & 3507 & 1753 & 584 & 292 & 146 & 19 \\
\hline 4 & 2021 & 3563 & 1781 & 594 & 297 & 148 & 20 \\
\hline 5 & 2022 & 3620 & 1810 & 603 & 302 & 151 & 20 \\
\hline
\end{tabular}

\subsubsection{Discusión}

En la presente investigación el universo de estudio constituye la demanda total, misma que es de 42226 familias, la demanda potencial que representa los posibles consumidores se obtuvo entre la demanda total y el porcentaje de aceptación del mercado el mismo que es del 88\% (ver cuadro 3) teniendo 37159 familias. El cálculo de la demanda insatisfecha se realizó entre al demanda potencial y la diferencia de la competencia (ver cuadro 7) siendo 8359 familias, las mismas que representan a los consumidores que no forman parte del mercado con los que la competencia trabaja, mientras que la demanda objetiva es de 6687 familias obtenidas de la demanda insatisfecha y el porcentaje de captación del mercado el mismo que es del 80\% (debido a que la mora es un producto consolidado en el mercado), la misma que representa los consumidores que se pretende captar de la demanda insatisfecha. De la demanda objetiva se deriva el consumo aparente por cliente (ver cuadro 12), el cual representa la cantidad de consumidores que posiblemente se captarán por cada periodo de tiempo: semestral, trimestral, mensual, quincenal, semanal y diario (mismo que representan 18 familias). Se puede concluir que existe un mercado potencial para los productores de mora de la parroquia El Altar.

\section{Conclusion}

- La mora en general tiene una gran aceptación en el mercado ya que alrededor de un $92 \%$ de las familias riobambeñas gustan del consumo de este fruto, en tanto la mora que se produce en la parroquia El Altar es apetecible para las familias de Riobamba, ya que según el análisis de la demanda indica que el 88\% consumiría dicho fruto, es así que los productores de la parroquia el Altar tienen grandes oportunidades de introducirse en el mercado.

- Los productores de mora de la parroquia El Altar tienen la posibilidad de ampliar su mercado, ya que al tratarse de un fruto ya consolidado en el mismo, se ha tomado un porcentaje de aceptación del mercado del $80 \%$, teniendo 6687 familias potenciales a las cuales podemos llegar con el producto anualmente, lo que representa un consumo aparente de 18 clientes 
diarios, sin duda alguna la mora tienen buenas perspectivas de ventas si se conserva la calidad y se ofrecen buenos precios, lo cual permitirá generar ingresos para los productores de mora aportando así a la disminución de la pobreza en el Altar.

- La mora desde un enfoque de comercialización es rentable para los vendedores, ya que al existir mercado los comercializadores tienen asegurada la venta al consumidor final, obteniendo una utilidad del 14\%, 42\% y 69\% cuando se vende la libra de mora a \$1, \$1,25 y \$1,50 respectivamente. Es así que productores y comercializadores de mora tienen amplio mercado por lo cual se constituye en ingresos económicos que le permita mejorar su calidad de vida.

\section{References:}

1. Ayala L., Valenzuela C., Bohórquez Y. (2013). “Caracterización fisicoquímica de mora de castilla (Rubus Glaucus Benth) en seis estados de madurez”. http://www.scielo.org.co/pdf/bsaa/v11n2/v11n2a02.pdf.

2. Castillo D., Quevedo L. (2016). “El Desarrollo Humano sostenible en los proyectos de Turismo Rural Comunitario”. http://www.eumed.net/rev/turydes/20/nariz-diablo.html.

3. Castillo D., Quevedo L. (2016). “Evaluación del desarrollo en los proyectos de turismo comunitario”. Editorial Académica Española. Página 46. https://www.eaepublishing.com/catalog/details//store/es/book/978-3-8417-51218/evaluaci\%C3\%B3n-del-desarrollo-en-los-proyectos-de-turismocomunitario.

4. Instituto Nacional de Estadísticas y Censos - INEC (2011). "Encuesta de Estratificación del Nivel Socioeconómico”. http://www.ecuadorencifras.gob.ec/encuesta-de-estratificacion-delnivel-socioeconomico/.

5. Instituto Nacional de Estadísticas y Censos - INEC (2010). "Censo de Población y Vivienda”. http://www.ecuadorencifras.gob.ec/banco-deinformacion/.

6. International Fund for Agricultural Development - IFAD (2001). “Informe Sobre la pobreza Rural 2001: El desafío consistente en acabar con la pobreza rural”. Fondo Internacional de Desarrollo Agrícola. Primera edición en inglés publicada por Oxford University Press (Reino Unido) en 2001. https:/www.ifad.org/documents/10180/6ec205c1-b70c-4e1f-b9da0a6a2ce4c79a.

7. Formichella M. \& Massigoge J. (2004). "El Concepto de Emprendimiento y su relación con la educación, el empleo y el 
desarrollo local”. https://www.researchgate.net/publication/281465619_El_concepto_d e_emprendimiento_y_su_relacion_con_el_empleo_la_educacion_y_e 1_desarrollo_local.

8. Klein Emilio (2012). "Políticas de mercado de trabajo y pobreza rural en América Latina". Food and Agriculture Organization of the United Nations - FAO. Viale delle Terme di Caracalla, 00153 Roma (Italia). http://www.fao.org/3/a-i2651s.pdf.

9. Ministerio de Agricultura, Ganadería, Acuacultura y Pesca - MAGAP (2013). "Manual de cultivo de mora". http://balcon.magap.gob.ec/mag01/magapaldia/HOMBRO\%20A\%20 HOMBRO/manuales/Manual\%20El\%20cultivo\%20de\%20la\%20\%2 0mora.pdf.

10. Morillo Diego (2011). "Respuesta del cultivo de mora Rubus Glaucus a la aplicación de dos tipos de bioles de frutas en dos dosis, Tumbaco, Pichincha”. https://www.researchgate.net/publication/277217820_Respuesta_del_ cultivo_de_mora_rubus_glaucus_a_la_aplicacion_de_dos_tipos_de_ bioles_de_frutas_en_dos_dosisTumbaco_Pichincha.

11. Spicker Paul (2009). "Definiciones de pobreza: doce grupos de significados”.

http://biblioteca.clacso.edu.ar/ar/libros/clacso/crop/glosario/06spicker .pdf.

12. Zegarra E., Tuesta J. (2009). "Boom Agrícola y persistencia de la pobreza rural". Food and Agriculture Organization of the United Nations - FAO. Viale delle Terme di Caracalla, 00153 Roma (Italia). http://www.fao.org/3/a-i0798s.pdf. 